\title{
Colour Representation in Lateral Geniculate Nucleus and Natural Colour Distributions
}

\author{
Naokazu Goda ${ }^{1,2}$, Kowa Koida ${ }^{1,2}$, and Hidehiko Komatsu ${ }^{1,2}$ \\ ${ }^{1}$ National Institute for Physiological Sciences, Okazaki 444-8585, Japan \\ ${ }^{2}$ Sokendai, Okazaki 444-8585, Japan \\ \{ngoda, koida, komatsu\}@nips.ac.jp
}

\begin{abstract}
We investigated the representation of a wide range of colours in the lateral geniculate nucleus (LGN) of macaque monkeys. We took an approach to reconstruct a colour space from responses of a population of neurons. We found that, in the derived colour space ('LGN colour space'), red and blue regions were compressed whereas purple region was expanded, compared with those in a linear cone-opponent colour space. We found that the expanding/compressing pattern in the LGN colour space was related to the colour histogram derived from a natural image database. Quantitative analysis showed that the response functions of the population of the neurons were nearly optimal according to the principle of 'minimizing errors in estimation of stimulus colour in the presence of response noise'. Our findings support the idea that the colour representation at the early neural processing stage is adapted for efficient coding of colour information in the natural environment.
\end{abstract}

Keywords: opponency, efficient coding, colour histogram.

\section{Introduction}

It is well established that signals encoded by three classes of cones (L, M and S) are combined at the retina to generate cone-opponent signals, which are then conveyed to the primary visual cortex via the lateral geniculate nucleus (LGN). The retinal and the LGN cone-opponent type of neurons are well modelled as a linear transformation of cone signals, L+M, L-M, and $\mathrm{S}-(\mathrm{L}+\mathrm{M})$ with appropriate weights. In this framework, colours can be represented by a three-dimensional space consisting of three orthogonal axes, each of which exclusively represents signal of the $\mathrm{L}+\mathrm{M}, \mathrm{L}-\mathrm{M}$ or $\mathrm{S}-(\mathrm{L}+\mathrm{M})$ mechanism $[1,2]$.

Although this colour space, which we call 'linear cone-opponent space', is useful to characterise physiological and psychophysical data in terms of colour mechanisms at the level of the retina and the LGN, it is well recognized that this colour space is not straightforwardly related to the CIELUV, CIELAB, or Munsell spaces, which are perceptually uniform. This suggests that colour signals are nonlinearly transformed into the perceptual colour representation at some neural level. Where does this transformation occur? Although it is likely that this nonlinear transformation involves computations at the cortical levels $[3,4]$, it is also possible that it begins at the subcortical level. 
Hanazawa et al. [5] investigated response properties of LGN colour-selective neurons in a macaque monkey, which is a good animal model of human colour vision, using a wide range of colour stimuli including highly saturated colours. They found that more than half of the neurons have response nonlinearities. These nonlinearities, most of which were compressive nonlinearity operating at the high-contrast range of colours, as well as variability of the response tuning among neurons, may explain complex, nonlinear characteristics observed at the perceptual level in humans.

In the present study, we revisited the colour representation in the LGN of the macaque monkey to explore the response nonlinearity at this neural level and its functional role. Here we took an approach to reconstruct a colour space from responses of a population of neurons $[6,7]$. We found that the derived colour space ('LGN colour space') was nonlinearly related to the linear cone-opponent colour space. Interestingly, the nonlinearity can partly explain why there are five basic hues in the Munsell colour space. This implies that the nonlinearity of the cone-opponent neurons at the subcortical level is involved in the transformation of cone signals into the perceptual representation. Furthermore, we found that the nonlinear encoding at this neural level was nearly optimal to natural colour distributions. These findings give important clues for understanding relationships among colour perception, neural responses, and natural colour statistics.

\section{Analysis of Colour Representation in LGN}

We analysed the responses of 38 LGN colour-selective neurons to 24 different chromaticities recorded from a macaque monkey performing a fixation task (see [5] for details). Figure 1a shows chromaticity of the stimuli in scaled and translated version of MacLeod-Boynton (MB) chromaticity diagram [1], in which horizontal axis exclusively represents L-M cone-opponent signals and vertical axis represents $\mathrm{S}-(\mathrm{L}+\mathrm{M})$ cone-opponent signals in a linear fashion. Here we call this space (and its linear transformation) 'linear cone-opponent space'. The stimulus was a stationary square of uniform colour and covering the entire receptive field on a dark grey background $\left(2.5 \mathrm{~cd} / \mathrm{m}^{2}, \mathrm{CIE} \mathrm{x}=0.310, \mathrm{y}=0.317\right)$. The luminance of the stimuli was held constant at $20 \mathrm{~cd} / \mathrm{m}^{2}$ or $7 \mathrm{~cd} / \mathrm{m}^{2}$. The stimuli were presented at least five times each for $500 \mathrm{~ms}$ in a pseudo-random order. The visual response was defined as the mean discharge rate during the stimulus presentation minus the baseline activity (200-0ms before stimulus presentation). The neurons were classified into three types based on the peak colour tuning direction in the MB chromaticity diagram; 19 L-M type neurons, 14 M-L type neurons, and $5 \mathrm{~S}-(\mathrm{L}+\mathrm{M})$ type neurons. There was no $(\mathrm{L}+\mathrm{M})-\mathrm{S}$ type of neuron.

\subsection{Reconstruction of Colour Space from Neural Data}

We applied classical multi-dimensional scaling (MDS) to the neural data to derive a uniform colour space, in which distances corresponded to pooled response differences of 38 LGN neurons. The pooled response difference between a pair of colours ('neural distance') was defined as Euclidian distance between 38-dimensional neural response vectors. The responses of each type of neurons were weighted by an inverse of the number of neurons assuming that there are equal numbers of these different types 
a

Stimulus chromaticities in cone-opponent space

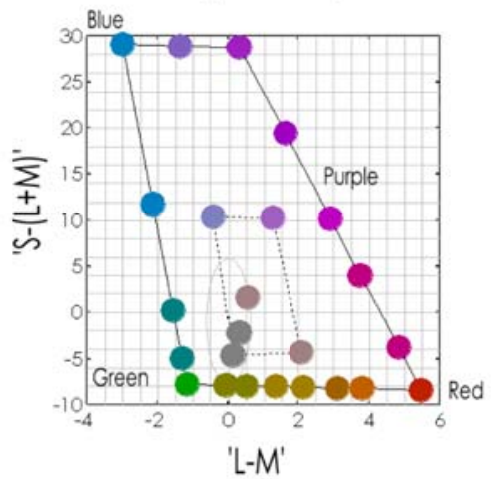

b

\section{LGN neuron-based color space}

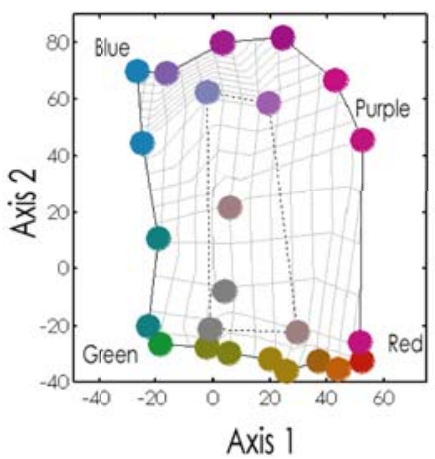

Fig. 1. (a) Colours used for the analysis plotted in the linear cone-opponent space (scaled and transformed MB chromaticity diagram. (b) Two-dimensional colour space reconstructed from responses of LGN neurons using a multi-dimensional scaling (LGN colour space). The two axes in the LGN colour space were aligned with those in the linear cone-opponent space.

of neurons in the LGN. For comparison with the linear cone-opponent space, the MDS-derived space was aligned to the linear cone-opponent space using a procrustes transformation (translation, reflection, orthogonal rotation, and scaling). Since the relative scale of the two axes of the linear cone-opponent space is unknown, we adjusted this relative scale so as to produce the best correspondence between the transformed MDS-derived space and the linear cone-opponent space.

Figure 1b shows the MDS-derived two-dimensional space, which well accounted for the neural distance data. Correlation coefficient between the distance in this space and the neural distance was quite high (0.998). Here we call the derived space 'LGN colour space'. In this LGN colour space, saturated blue and red regions were compressed compared with the linear cone-opponent space. This clearly shows that cone signals are nonlinearly transformed at this neural level.

Interestingly, purple region was relatively expanded in the LGN colour space; purple colour was located around midpoint between red and blue in the linear coneopponent space, whereas it was located near upper right corner in the LGN colour space. The distance between purple and white was comparable with that between blue and white in the LGN colour space. Thus, purple is one of salient colours at the LGN level. This is an interesting characteristic because purple is one of basic hues in the Munsell colour space (red, green, yellow, blue and purple, which are equally spaced in the space). This suggests that the nonlinear transformations into the perceptual colour representation may begin at this neural level.

\subsection{Model of LGN Colour Space}

How is the LGN colour space related to the linear cone-opponent space? We hypothesized that (1) the LGN colour space is comprised of independent cone-opponent L-M axis and $\mathrm{S}-(\mathrm{L}+\mathrm{M})$ axis, but (2) there are simple compressive nonlinearity (saturation) 

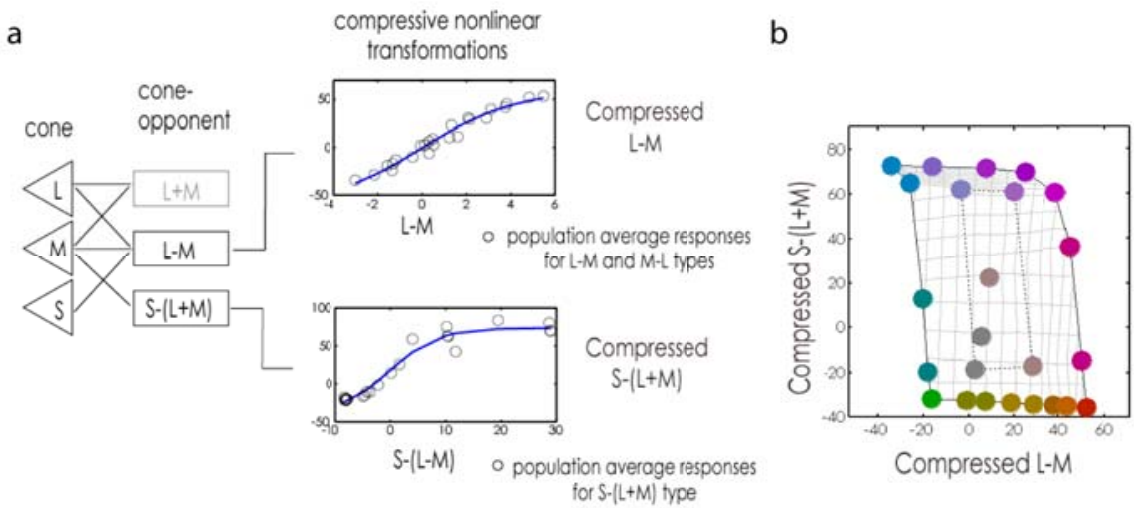

Fig. 2. (a) Population average of the neural responses along the two axes (open circle) and the modelled response functions (continuous line). For the L-M axis, responses of the M-L types of neurons were averaged with those of the L-M type of neurons after inverting the sign. (b) A two-dimensional space reconstructed by the model.

along each of the two axes. Next, we examined how this simple model can account for the LGN colour space.

Figure $2 \mathrm{a}$ shows response functions along the two axes and the two-dimensional colour space reconstructed by the model. The response function of each axis was modelled by the sigmoid functions (hyperbolic tangent functions) [5]. The modelled two-dimensional colour space could replicate the pattern of the LGN colour space (fig. 2b), although there were still distortions that could not be explained by the model. The distances in the modelled LGN colour space was more highly correlated with the neural distances than those in the linear space. Thus the pattern in the LGN colour space is at least partly explained by considering simple saturation of the two axes responses, although other complex nonlinearity may be involved as well.

\section{Relationships between LGN Colour Representation and Natural Colour Distributions}

What role does the nonlinearity plays? One hypothesis is that the nonlinear transformation is related to an optimal encoding of colours in the natural environment. Computational studies have suggested that the colour mechanisms at the retina and the LGN levels are adapted to transmit colour information in the natural environment through optic nerve fibre with limited capacity $[8,9]$. The compressive nonlinearity of the response functions can also be interpreted computationally in terms of an optimal encoding of natural inputs $[10,11]$. To test this hypothesis, we examined how the LGN colour representation is related to natural colour distributions.

\subsection{Analysis of Natural Colour Distributions}

Histogram of natural colours were evaluated by using 327 natural scene images from McGill calibrated colour image database [12], which is a collection of calibrated 
a

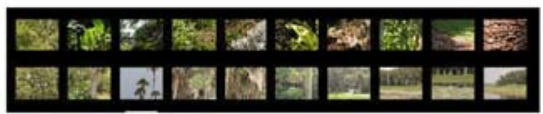

b

\section{Histogram of Natural Colors in cone-opponent space}

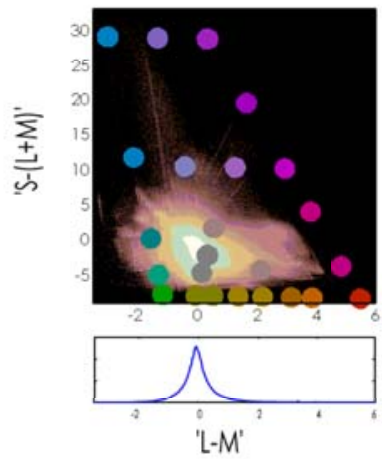

Histogram of Natural Colors in LGN color space

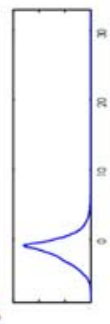

marginal cistribution
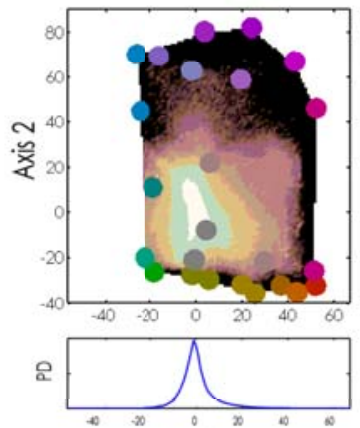

Axis 1

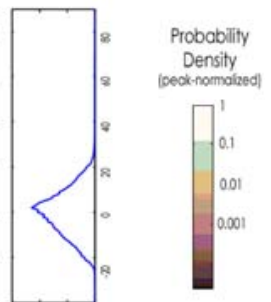

Fig. 3. (a). Example of natural images. (b) Histogram of natural colours evaluated using the natural image database. Colour density is shown by pseude-colour scale in the linear coneopponent space (left) and in the LGN colour space (right). Marginal distributions are also shown along each axis.

natural images including plants, landscape and so on (fig. 3a). The images were preprocessed taking light adaptation at photoreceptor level into account. We applied von Kries scaling to the LMS cone excitations for each image [13]; this adjusts the gains of LMS cone excitations independently so that mean luminance and chromaticity over entire scene is constant (unit luminance of illuminant $\mathrm{C}$ ). Then we computed twodimensional colour histograms in the linear cone-opponent space as well as in the LGN colour space using all images.

Figure $3 \mathrm{~b}$ shows derived colour histograms plotted in the linear cone-opponent space and the LGN colour space. Natural colours were highly concentrated around the white point in the linear cone-opponent space (fig. $3 \mathrm{~b}$ left), whereas they were more flatly distributed in the LGN colour space (fig. $3 \mathrm{~b}$ right). Importantly, the compressed region in the LGN colour space (saturated blue and red) corresponded to the lowdensity regions in the linear cone-opponent space. This trend supports the hypothesis that the compressive nonlinearity is related to natural colour distributions.

\subsection{Are the Response Functions Optimal for Natural Colour Distributions?}

To examine whether the LGN colour representation is optimised for natural colour distributions more quantitatively, we asked whether the response characteristics of the LGN neurons are optimal in terms of optimisation theories. Assuming that the two axes in the LGN colour space are orthogonal, we analysed the response function along each axis based on two theories. One is the 'Pleistochrome' theory constructed by von der Twer and MacLeod [11]. According to this theory, the optimal response 
function $\mathrm{g}(\mathrm{x})$, is derived from the probability density function of the input, $\mathrm{p}(\mathrm{x})$, by eq. 1. This function minimizes the error of estimation of input signal (e.g., chromaticity) from the output (response) in the presence of output noise. Another more popular theory is the 'Infomax' developed by Laughlin [10] and recently used by Long et al. [14] for investigating relationships between colour perception and natural colour statistics. According to this theory, the optimal response function is derived by the cumulative probability density function (eq. 2). In both cases, the optimal response functions are derived if the input distributions are given.

$$
\begin{gathered}
g(x)=\int_{-\infty}^{x} p(u)^{1 / 3} d u \\
g(x)=\int_{-\infty}^{x} p(u) d u
\end{gathered}
$$

Figure 4 shows the response functions optimal to the distributions of the L-M and S$(\mathrm{L}+\mathrm{M})$ cone-opponent signals predicted from the two theories. The functions predicted from the 'Pleistochrome' theory fitted well to the neural response data for both axes. These are also close to the response function of the LGN colour space model (fig.2a). On the other hand, the functions predicted from the 'Infomax' were steeper than the neural response functions. These suggest that the colour mechanisms at the LGN level are nearly optimal to natural colour distributions, according to the principle of minimizing the error for estimating colour from the responses.

Contrary to our results, von der Twer and MacLeod [11] did not find evidence that the chromatic response functions of the LGN neurons were optimal to natural colour statistics. The discrepancy between their results and ours may be due to differences in the image database used for evaluating natural colour distributions. They used images collected by Ruderman et al. [15], which included only limited classes of natural scenes. The colours in these images are more heavily concentrated around the white

a

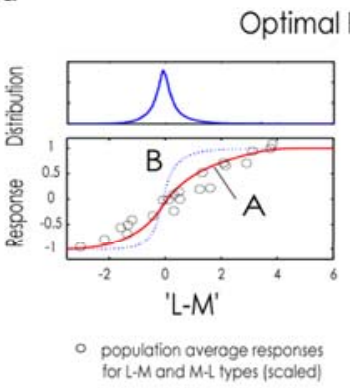

Optimal Response Functions

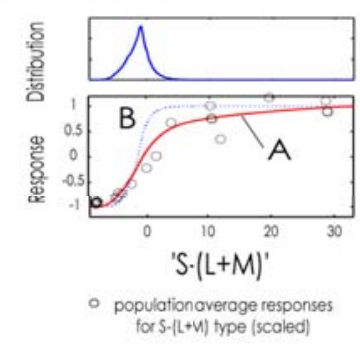

b Optimal Response Functions A

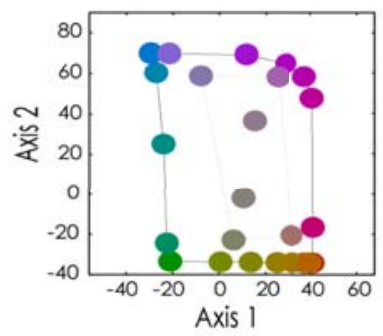

Fig. 4. (a) Comparisons between the response functions of the LGN neurons (open circle) and the optimal response functions predicted from the 'Pleistochrome' theory (continuous line, A) and the 'Infomax' theory (dotted line, B) along L-M axis (left) and S-(L+M) axis (right). Upper panels show natural colour distributions along each axis. (b) A two-dimensional space composed of optimal response functions predicted from the 'Pleistochrome' theory. 
point than those in the database that we used. Thus, the density of highly saturated colours should be underestimated if that database was used.

Note also that we computed colour distributions after applying von Kries adaptation. Without von Kries adaptation, the colour distribution becomes flatter and the optimal response functions for those distributions will become more linear than the neural response functions. This means that receptor gain control, as well as compressive nonlinearity, has an important role in efficient coding of colours.

\section{Conclusion}

We reconstructed a colour space from responses of a population of LGN neurons. We found that the derived colour space ('LGN colour space') was considerably expanded/compressed compared with those in the linear cone-opponent colour space. Interestingly, the expanding/compressing pattern may partly explain emergence of five basic hues in colour appearance. This suggests that nonlinear transformations into the perceptual colour representation may begin at this early neural processing stage. Furthermore, we found that the expanding/compressing pattern in the LGN colour space can be explained simple compressive nonlinearity of the cone-opponent type of neurons. Such nonlinear response characteristics were nearly optimal to natural colour distributions according to the principle of 'minimizing errors in estimation of stimulus colour in the presence of response noise'. This supports the idea that the colour representation at the early neural processing stage is adapted for efficient coding of colour information in the natural environment.

\section{References}

1. MacLeod, D.I.A., Boynton, R.M.: Chromaticity Diagram Showing Cone Excitation by Stimuli of Equal Luminance. J. Opt. Soc. Am. 69, 1183-1186 (1978)

2. Derrington, A.M., Krauskopf, J., Lennie, P.: Chromatic Mechanism in Lateral Geniculate Nucleus of Macaque. J. Physiol. 357, 241-265 (1984)

3. De Valois, R.L., Cottaris, N.P., Elfar, S.D., Mahon, L.E., Wilson, J.A.: Some Transformations of Color Information from Lateral Geniculate Nucleus to Striate Cortex. Proc. Natl. Acad. Sci. U.S.A. 97, 4997-5002 (2000)

4. Stoughton, C.M., Conway, B.R.: Neural Basis for Unique Hues. Curr. Biol. 18, R698R699 (2008)

5. Hanazawa, A., Komatsu, H., Murakami, I.: Neural Selectivity for Hue and Saturation of Colour in the Primary Visual Cortex of the Monkey. Eur. J. Neurosci. 12, 1753-1763 (2000)

6. Valberg, A., Seim, T., Lee, B.B., Tryti, J.: Reconstruction of Equidistant Color Space from Responses of Visual Neurons of Macaque. J. Opt. Soc. Am. A 3, 1726-1734 (1986)

7. Young, R.A.: Principal-component Analysis of Macaque Lateral Geniculate Nucleus Chromatic Data. J. Opt. Soc. Am. A 3, 1735-1742 (1986)

8. Buchsbaum, G., Gottschalk, A.: Trichromacy, Opponent Colours Coding and Optimum Colour Information Transmission in the Retina. Proc. Roy. Soc. Lond. B 220, 1934-1990 (1983) 
9. Attick, J.J., Li, Z., Redlich, A.N.: Understanding Retinal Color Coding from First Principles. Neural Comp. 4, 559-572 (1992)

10. Laughlin, S.B.: A Simple Coding Procedure Enhances a Neuron's Information Capacity. Z. Naturforsch. 36(c), 910-912 (1981)

11. von der Twer, T., MacLeod, D.I.: Optimal Nonlinear Codes for the Perception of Natural Colours. Network 12, 395-407 (2001)

12. Olmos, A., Kingdom, F.F.A.: McGill Calibrated Colour Image Database, http: //tabby.vision.mcgill.ca

13. Webster, M.A., Mollon, J.D.: Adaptation and the Color Statistics of Natural Images. Vision Res. 37, 3283-3298 (1997)

14. Long, F., Yang, Z., Purves, D.: Spectral Statistics in Natural Scenes Predict Hue, Saturation, and Brightness. Proc. Natl. Acad. Sci. U.S.A. 103, 6013-6018 (2006)

15. Ruderman, D.L., Cronin, T.W., Chiao, C.: Statistics of Cone Responses to Natural Images: Implication for Visual Coding. J. Opt. Soc. Am. A 15, 2036-2045 (1998) 